\title{
EPIDEMIOLOGICAL GIS: Understanding Emerging Critical Issues
}

\author{
Josh U. Ogbonna \\ Department of Geography and Planning, Abia State University, Uturu. Nigeria
}

\begin{abstract}
This paperexamines the roles of GIS in epidemiological research, andidentifies needs for spatial epidemiology. It argues that the objectivefunction of spatial epidemiology is to identify patterns and correlates it to geospecific variation in health risks incidences. GISsupports disease mapping, location analysis, the characterization of populations, spatial statistics and simulation modeling. Despite these flurries of applications, they are yet to sufficiently identify disease causes and correlates.This is partly because of the failure of GIS to provide tools appropriate forepidemiological research and analysis. Hence, the need to review our understanding towards the relationship between causes of a disease and its spatio-temporal information. Thus the need for a design and implementation of a space-time information systems and process-based disease models for epidemiology
\end{abstract}

Keywo rds GIS, GPS, DSS, Epigis, Epidemiology, Spatial Statistics, Spatial Models, Geostatistics, Spatio-Temporal

\section{Introduction}

According to[1], the issue of public health concerns the knowledge, monitoring, assessment and maintenanceof the health of human populations. This involves monitoringdisease outcomes, through the identification, designing and implementinginterventions to ameliorate health risk factors. GIS is making substantive contributions in public health at a hands-on level. Logical questions such as 'Where is the best place for the newclinic?', 'Why are disease mortality high?', 'Why are there a disease cluster?', 'Where are the source point of this disease?', are allgeospecific questions that can be addressed using spatial statistical techniques and GIS[2].

As epidemiology is the study of health and disease in human populations, and, because populations are inextricably bound to 'place', it seems reasonableto expect GIS to advance Epidemiology as Geosciences[3]. Despite the manypractical applications in public health, some authors believe this expectationhasn't been fully met as shown in[4]. Sources for this failure are several andinclude the lack of trained epidemiologists to 'think spatially'; a lack of epidemiological studies that invoke GIS applications/concepts as a Decision Support System (DSS) [5]; and the failure of commercial on-the-shelf (COTS) GIS toprovide appropriate tools for spatial ep ide miology[6]. As a tool for advancingepidemiology as science, GIS thus

* Corresponding author:

juogbonna@yahoo.com (Josh U. Ogbonna)

Published online at http://journal.sapub.org/ ajgis

Copyright (C) 2012 Scientific \& Academic Publishing. All Rights Reserved appears to have severe limitations. To understand these limitations, this paperconsiders GIS both as technology and as a scientific tool.

\section{GIS and Spatial Analysis Concepts in Epidemiology}

The issues ofIntelligent Maps (iMaps)

Visualization is one of the first steps in exp loratory spatial data analysis[7]. GIS creates intelligent maps (iMaps) that are digital, interactive, queriable, and can be subjected to Boolean operations for morbidity and mortality patterns in relation to population density, putative exposures and geo-based features. It therefore constitutes a lin $\mathrm{k}$ between statistical plots, quantitative appraisals and geographic maps[5],[8],[9] forming the geostatistical (choropleth/density) maps. Geostatistics is a methodology for incorporating the spatial and temporal coordinates of observations in data processing. However, the key feature of epidemiological data is their location and display in a space-time continuum which should be incorporated in any descriptive or quantitative analys is of the data[10]. Recently, GIS has emerged as an innovative and important component of many projects in public health and epidemiology. GIS can be used to map and analyze the geographical distribution of population at risk, health outcomes and risk factors; to explore as sociations between risk factors and health status; and to plan public health services[11]. As it is traditional, the adaptation of mapping and cartographic techniques in search for answers begins with the formulation of spatio-epidemio logic hypothesis.

Location-AllocationProblem 
The location-allocation problem addresses the need toplace health facilities and services in 'the best' geographic locations as to optimize its objective functions (minimizing cost and maximizing accessibility) through the identification of the best possible location. It provides solution for initial facility problem and/or the incremental facility problem. GIS and its add-ons support the determination of optimal facility location or where health facilities are best located in relation to its users; through the creation of attribute layers for an overlay and split operations, the estimation of ambulance travel times to and from hospitals and ambulance routing path, theidentification of hospital catchment areas, and the determination of the hospitals niche population. In public health, GIS has provencontributions in vector control (e.g. where to place the intervention?) to the impact (why the intervention?) to reducemalaria, and other vector-borne diseases.

Characterization of populations.

To outsiders, epidemiologis ts seem fixatedon 'nume rators' and 'denominators', and with good reason: In a disease rate,the numerator is the number of the disease cases while the denominator is the population at riskof contracting the disease. In spatial epidemiology, the characterization ofgeographic populations is an important task directly supported by GIS. This is because GIS is interested in giving answer to where, what is where and why. Thisentails exposure ass essments involving the identificationof high-risk populations, the interplay of factors among the attributes and whyrisks incidences are high.

Spatial statistics.

Spatial statistics quantify geographic variation in geographicvariables. They can identify violations of assumptions of independencerequired by many epidemiological statistics; and measure how populations, their characteristics, covariates and risk factors vary in geographic space[12],[13], and[5]. Methodological researches on disease cluster techniques for spatial epidemiology are indispensible for advancing spatial epidemiological science.

Spatial models.

Recent developments of spatial models in public healthaccording to[14] include Bayesian smoothing of disease rates, geostatisticalmodels, and Haggastrand Mean Information Field (MIF) for the Monte Carlo simulation estimation techniques. Thesemodels address important is sues of the stabilization of disease rates, interpolation, map presentation, information flow, contagion rates and the estimation of variables at 'not gauged'locations.

While these models are formidable, they support descriptiveepidemiology and do not directly increase our ability to link humanhealth outcomes to putative exposures and environmental risk factors. At best they can identify a local excess of disease that maybe suggestive usinggeographic proximity as a surrogate for a possible cause. This seems poorpayoff for what is often a costly and time consuming geographic investigation.

Spatial Data
Spatial data is information about the location and shapes of geographic features, in the form of either vector or raster. These features are created as graphic maps in three geometric forms as points, lines, and polygons (areas). Associated with these graphic data are data records that provide the identifying and descriptive data attributes. An outcome from this enterprise re inforces the where answer to epidemiological data.

\section{Needs of Spatial Epidemiology}

This lack of scientific payoff is rooted in technological determinis $m$ whereby GIS tools determine the hypotheses that can be addressed. The questions one canask of the data depend implicitly on the data models, spatial data structures, and Boolean queries employed by the GIS. The adaptation of health data unto geographic virtual models explains the process-pattern link governing spatial epidemiological research.

Process-pattern link.

We need an increased understanding of the relationshipsbetween disease processes and the resulting disease patterns. Spatial dis eas epatterns are the outcomes of space-time processes, and several alternativeprocesses may give rise to similar spatial patterns[15] makingit very difficult to interpret spatial disease patterns in other than broadly descriptiveterms. The trajectory of this link requires a clear understanding of the models, the data and the data's dynamic process while the estimation of such a model's parameters requiresspace-time information systems.

Models of process.

Models of process are expressed in terms of the physicaland biological mechanisms underlying the system under study. This contrastswith models of data that are expressed in terms of the data's statistical properties.At present most if not all spatial models in public health are models ofdata, rather than models of process. While useful for pred iction, the para meters of models of data have limited epidemiological utility. In contrast, theparameters of process models are by definition directly interpretable in terms of underlying diseases. Compartmental analysis according to[16] is a powerfulapproach for constructing models of space-time processes that holds greatpromise in epidemiology.

Space-time information systems (STIS)

Estimation of the parameters ofsuch process models will require information systems capable of dealing with GIS in epidemiology usingspatial and temporal referencing. While several authors recognize the need fortime GIS' in order to represent space-time data in general (e.g.[17],[18]) and human disease data in particular[19], it's essentialrole in the estimation of parameters of space-time process models hasreceived little recognition. Because STIS will make possible estimation of theparameters of space-time models, they are expected to be indispensible foradvancing spatial epidemiologic science. 
According to[20] and[5], in designing statistics for spatial epidemiology certain data attributes must be considered. These are:

- The spatial coordinates of the data noting their proximity measurements (determining their nearest neighbor index, and accessibility index),

- An understanding of their point - pattern display (determin ing their Variance-Mean-Ratio), and

- Identifying certain data nominal attributes such as relevant descriptors of place,including its spatial location, exposure and covariates.

It must be noted that for diseases with multiple casual factors an integrating approach is essential. GIS techniques can assess a multiplicity of external factors, such as physical variables, technological hazards and social/demographic factors[21]. More so, it is also desirable to integrate biological indicators into exposure assessment, with respect to the timing of exposures, induction and latency.

\section{Discussion and Conclusions}

The above presentation has beento stimulate thoughts onobserved problems in spatial epidemiological modeling and on the inventing technologies requiredto solve them. In that, a problem solution stimulates the development ofcritical technology; and secondly, a technologicaladvancement that results to problems that were not thetarget of the innovation,[4]. The GIS adaptation in spatial epidemiology fell within the second group. This problem is more exposed in the non-availability of reliable exposure data. In most cases, the use of calculated or measured data is, at best, only an approximation of the actual exposure. Data on past exposure and location has to be detailed and accurate, concerning mobility and other outcomes that are dependent on time variation. The results from analyses on regional data will of course depend on the spatial scale used. This sophistication display possibilities that can bring a false sense of accuracy. Noting that results from analys es can never be more accurate than the quality of the data captured[22],[23] and[24].

Within the last 3 years, Nigeria has experienced some catastrophic events that clearly provide evidence of the importance of a spatially-inducedhealth information system.The September, 1998 C130 plane crash; May 2002 EAS airline crash; October, 2005 Bellview plane crash; Dece mber, 2005 Sosoliso crash; 2006 ADC airline and June 2012 DANA crash and the associated health hazard has exposed the inappropriate management of post-plane crash era. Thus provides an enormous evidence of the need for a spatially-induced digital platform as a decision support in the post emergency management efforts. Lack of a functional EpiGIS platform in Nigeria has militatedagainst efficient and sustainable post disaster management techniques. With constant outbreak of meningit is in the Northern part of Nigeria and severe water pollution in the south causing disastrous outbreak of typhoid presents strategic failures in epidemiological post-cris is management.

It is the view of this paper that there is an important need to carefully design appropriate GIS technology for epidemiological research and management; and adaptations that will lead to the evolution of Spatial Decision Support System(SDSS) in public health which will eventually drive the database engine for the health system. This system will provide centralized access tonational health anddisease database and a repository of GIS epidemiological tools. These will result to:

An increased understanding of the relationships between health status, disease processes and patterns;

Evolution of process-based disease and health models;

- Understanding space-time information systems for epidemiology; and

Development of statistical models for spatio-temporal epidemiological indices.

This paper should be seen as a contribution to the flurry of papers on spatial epidemiology, and concludes that sustainable research on epidemiology must recognize the spatio-temporal information on diseases. The model arising from this situation must be able to calibrate space-time data on health outcomes into epidemiologically meaningful measures. The geo-specificity of the health database constitutes the foundation of Epidemiological Geographic Information Systems (EpiGIS).

\section{REFERENCES}

[1] Thacker SB, Stroup DF et al. (1996) Surveillance in environmental public health: Issues, systems and sources. American Journal of Public Health 86(5):633-638

[2] Gatrell AC, Loytonen M (2010) GIS and health research: An introduction. In: Gatrell A.C. and Loytonen M. GIS andhealth. Tay lor and Francis, London, pp 47-59

[3] Jacquez GM (1998) GIS as an Enabling Technology. In: Gatrell AC, Loytonen M, GIS and health. Tay lor and Francis, London, pp 17-28

[4] JacquezG.M(2010) Spatial analy sis in epidemiology: Nascent science or a failure of GIS?

[5] Ogbonna, J. U (2009) Benefits of GIS and related technologies in the management of health care systems for Nigeria. Nigerian Journal for Development Research. $1(1): 34-40$

[6] Jacquez GM, Grimson R et al. (1996) The analy sis of disease clusters Part II: Introduction to techniques. Infection Control and Hospital Epidemiology 17:385-397

[7] Jacquez GM. (1999) Disease clustering for uncertain locations. In: Lawson A, Bertollini R, Disease mapping and risk assessment for public health decision making. Wiley, London

[8] Monmonier M (1996) How to lie with maps. The University of Chicago Press, Chicago, IL 
[9] Tweedie L (1997) Characterizing interactive externalizations. CHI'97, ACM Press, Atlanta, GA

[10] Loytonen M (2010) GIS, time geography and health. In: Gatrell AC, Loytonen M, GIS and health. Tay lor and Francis, London.

[11] Bailey TC, Gatrell AC (1995) Interactive spatial data analy sis. Longman Scientific \& Technical, Essex, England

[12] Rushton G, Lolonis P (1996) Exploratory spatial analysis of birth defects in an urban population. Statistics in Medicine $15: 717-726$

[13] Haining R (1998) Spatial statistics and the analysis of health data. In: Gatrell AC, Loytonen M, GIS and health. Taylor and Francis, London, pp 29-48

[14] Devine OJ, Lou is TA et al. (2000) Empirical Bay es estimators for spatially correlated incidence rates. Environmetrics 5:381-398

[15] Schaerstrom A (1996) Pathogenic paths? A time geographical approach in medical geography. Lund University Press, Lund, Sweden

[16] Jacquez JA (1996) Compartmental analysis in biology and medicine. BioMedware, Ann Arbor, MI.

[17] Langran G (2010) Time in Geographic Information Systems.
Taylor and Francis, London

[18] Peuquet DJ (1994) It's about time: A conceptual framework for the representation of temporal dynamics in GIS. Annals of the Association of American Geographers 84(3):441-461

[19] Loytonen M, GIS and health. Tay lor and Francis, London, pp 3-16

[20] Jacquez G.M, Waller LA et al. (1996) The analy sis of disease clusters Part I: State of the art. Infection Control and Hospital Epidemiology 17:319-327

[21] Hjalmars U (2000) Environmental epidemiology and GIS. Commentary on "GIS in Public and Environmental Health: Visualization, Exploration and Modelling by Anthony Gatrell

[22] Gatrell, A.C. and Löytönen, M. (1998, eds) GIS and Health, Taylor and Francis, London.

[23] Vine, M .F., Degnan, D and Hanchette, C. (1997) Geographic information systems: their use in environmental epidemiologic research, Environmetal Health Perspectives;105:598-605.

[24] Waller, L.A. (1996) Epidemiologic uses of geographic information systems (GIS), Statistics in Epidemiology Report: Spring/Summer 1996. 